\title{
Concerning Sets of the First Baire Category with Respect to Different Metrics
}

\author{
by \\ Maria MOSZYŃSKA and Grzegorz SÓJKA \\ Presented by Czesław BESSAGA
}

\begin{abstract}
Summary. We prove that if $\varrho_{H}$ and $\delta$ are the Hausdorff metric and the radial metric on the space $\mathcal{S}^{n}$ of star bodies in $\mathbb{R}^{n}$, with 0 in the kernel and with radial function positive and continuous, then a family $\mathcal{A} \subset \mathcal{S}^{n}$ that is meager with respect to $\varrho_{H}$ need not be meager with respect to $\delta$. Further, we show that both the family of fractal star bodies and its complement are dense in $\mathcal{S}^{n}$ with respect to $\delta$.
\end{abstract}

0. Introduction. Following [3], we say that a set $A$ in a metric space is of the first Baire category or meager if $A$ is a countable union of nowhere dense sets; the complement of a meager set is called generic and its elements typical.

The problem of what families are of the first Baire category in the hyperspace $\mathcal{C}^{n}$ of nonempty compact subsets of $\mathbb{R}^{n}$ endowed with the Hausdorff metric $\varrho_{H}$ or in subspaces of $\mathcal{C}^{n}$ has been considered by many authors; see, for example, [3], [4], [10].

The present paper is an effect of discussion around the paper [1] concerning Baire category problems for some families of compact $n$-dimensional manifolds with boundary in $\mathbb{R}^{n}$. Some of those families coincide with $\mathcal{S}_{\varepsilon}^{n}$ for $\varepsilon>0$, where $\mathcal{S}_{\varepsilon}^{n}$ consists of the compact subsets of $\mathbb{R}^{n}$ star-shaped at 0 , with continuous radial functions, and containing the ball $\varepsilon B^{n}$. In view of the main result, the Frame Approximation Theorem ([1, p. 536]), the subfamily of $\mathcal{S}_{\varepsilon}^{n}$ whose members have fractal boundaries is meager. The proof is based on Theorem 2 in [3] that concerns the Hausdorff metric, but the author deals

2010 Mathematics Subject Classification: 54E52, 52A30, 28A80.

Key words and phrases: sets of the first Baire category, star body, fractal dimension, fractal star body, locally fractal star body. 
with the sup metric as well. For the particular case mentioned above this metric corresponds to the radial metric.

However, it is well known that the radial metric is topologically strictly stronger than the Hausdorff metric (see [5] or [8, 14.3.4]).

Generally, it is easy to see that if $d_{1}$ and $d_{2}$ are two metrics on some $X$ and $d_{2}$ is topologically stronger than $d_{1}$, then a subset $A$ of $X$ may be meager in $\left(X, d_{1}\right)$ but nonmeager in $\left(X, d_{2}\right)$. (For instance, if $d_{1}$ is the Euclidean metric and $d_{2}$ the "railway metric" in $\mathbb{R}^{2}$, then for $x \neq 0$, the segment $A=\Delta(0, x)$ is meager in $\left(\mathbb{R}^{2}, d_{1}\right)$ but not meager in $\left(\mathbb{R}^{2}, d_{2}\right)$.)

Thus, it is natural to ask the following question concerning the family $\mathcal{S}^{n}:=\bigcup_{\varepsilon>0} \mathcal{S}_{\varepsilon}^{n}$ :

Question 0.1. Is it true that for any subfamily $\mathcal{A}$ of $\mathcal{S}^{n}$, if $\mathcal{A}$ is meager in $\mathcal{S}^{n}$ with respect to $\varrho_{H}$, then it is meager in $\mathcal{S}^{n}$ with respect to $\delta$ ?

We answer this question in the negative in Section 2.

Let us notice that for star bodies the radial metric is the most natural and commonly used. Thus, we are interested in the following.

Question 0.2. Does the Frame Approximation Theorem of [1] hold for the families $\mathcal{S}_{\varepsilon}^{n}$ or their union, $\mathcal{S}^{n}$, endowed with the radial metric?

This problem is still open. In Section 3 we prove some related results on the family of nonfractal star bodies and some families of fractal star bodies (Corollaries 3.8 and 3.9).

1. Preliminaries. We follow, in principle, the terminology and notation used, for instance, in [7]. In particular, $\operatorname{dim} X$ is the topological dimension of a (separable) metric space $X$ and $\operatorname{dim}_{F}$ is a fractal dimension (as defined axiomatically in [7]). The Hausdorff and the Minkowski dimensions are denoted by $\operatorname{dim}_{H}$ and $\operatorname{dim}_{M}$, respectively.

The unit ball in $\mathbb{R}^{n}$ with Euclidean metric $d_{E}$ is denoted by $B^{n}$.

We use the symbols cl, int, bd, and conv (with subscripts, if needed) to denote closure, interior, boundary, and convex hull, respectively.

The segment with endpoints $a, b \in \mathbb{R}^{n}$ is $\Delta(a, b)=\{(1-t) a+t b \mid 0 \leq$ $t \leq 1\}$. The unit sphere in $\mathbb{R}^{n}$ in $S^{n-1}:=\operatorname{bd} B^{n}$.

A subset $A$ of $\mathbb{R}^{n}$ is star-shaped at $a \in A$ provided that $\Delta(a, x) \subset A$ for every $x \in A$. The set

$$
\text { ker } A:=\{a \in A \mid A \text { is star-shaped at } a\}
$$

is called the kernel of $A$. A star-shaped set $A$ is called a star body whenever $A$ is compact and $\operatorname{cl} \operatorname{int} A=A$. 
We are interested in the family $\mathcal{S}^{n}$ of star bodies in $\mathbb{R}^{n}$ with 0 in the kernel. It can be endowed with the Hausdorff metric $\varrho_{H}$, but the most natural metric for $\mathcal{S}^{n}$ is the radial metric $\delta$ defined by the radial functions restricted to $S^{n-1}$ : for every $A \in \mathcal{S}^{n}$ the radial function $\rho_{A}: S^{n-1} \rightarrow \mathbb{R}_{+}$is defined by

$$
\rho_{A}(u):=\sup \{\lambda>0 \mid \lambda u \in A\} ;
$$

and the radial metric $\delta$ is just the sup metric for radial functions.

As is well known, the $\varepsilon$-parallel body $(A)_{\varepsilon}$ of a compact subset $A$ of a metric space is defined to be the set of points with distance at most $\varepsilon$ from $A$. In particular, in the Euclidean $n$-space,

$$
(A)_{\varepsilon}=A+\varepsilon B^{n},
$$

where + is the Minkowski addition.

While the Minkowski addition has especially good properties for compact convex sets (e.g. the cancellation law is satisfied), for star bodies the so called radial addition $\tilde{+}$ is more natural and commonly used. It can be defined by means of radial functions as follows:

$$
\rho_{A_{1} \tilde{+} A_{2}}(u):=\rho_{A_{1}}(u)+\rho_{A_{2}}(u) \quad \text { for every } u \in S^{n-1} .
$$

Then the counterpart of the formula (1.1) for the radial $\varepsilon$-hull has the radial sum $A \tilde{+} \varepsilon B^{n}$ on its right hand side.

A star body $A$ in $\mathbb{R}^{n}$ is fractal with respect to a fractal dimension $\operatorname{dim}_{F}$ if its boundary is fractal with respect to $\operatorname{dim}_{F}$, that is, $\operatorname{dim}_{F} \mathrm{bd} A>n-1$, or equivalently, $\operatorname{dim}_{F} \operatorname{graph} \rho_{A}>n-1$. A star body $A$ is locally fractal if for every nonempty $U$ open in bd $A$ the set $U$ is fractal (compare [7]).

2. Baire category problem for star bodies. The following result gives a negative answer to Question 0.1.

THeOREM 2.1. There exists a family $\mathcal{A}$ in $\mathcal{S}^{n}$ meager with respect to $\varrho_{H}$ but not meager with respect to $\delta$.

Proof. Let $\mathcal{A}$ be the ball in the hyperspace $\left(\mathcal{S}^{n}, \delta\right)$, with center $B^{n}$ and radius $r=1 / 3$. That is,

$$
\mathcal{A}:=\left\{A \in \mathcal{S}^{n} \mid \delta\left(A, B^{n}\right) \leq 1 / 3\right\} .
$$

In other words,

$$
A \in \mathcal{A} \Leftrightarrow A \in \mathcal{S}^{n} \text { and } \frac{2}{3} B^{n} \subset A \subset \frac{4}{3} B^{n} .
$$

Of course, $\operatorname{int}_{\left(\mathcal{S}^{n}, \delta\right)} \mathcal{A} \neq \emptyset$, whence $\mathcal{A}$ is not meager with respect to $\delta$, because $\left(\mathcal{S}^{n}, \delta\right)$ is a Baire space.

We are going to prove that $\mathcal{A}$ is meager with respect to $\varrho_{H}$.

Let us first show that

$$
\operatorname{int}_{\left(\mathcal{S}^{n}, \varrho_{H}\right)} \mathcal{A}=\emptyset,
$$


i.e., for any $A \in \mathcal{A}$ there exists a sequence $\left(X_{k}\right)_{k \in \mathbb{N}}$ in $\mathcal{S}^{n} \backslash \mathcal{A}$ convergent to $A$ with respect to $\varrho_{H}$.

Take an $A \in \mathcal{A}$. Consider a sequence $\left(\alpha_{k}\right)_{k \in \mathbb{N}}$ in $(0 ; \pi / 4]$ convergent to 0 , and let $a=\frac{1}{3} e_{n}$. For every $k$, let $C_{k}$ be the cone in $\mathbb{R}^{n}$ defined by

$$
C_{k}:=\{a\} \cup\left\{x \in \mathbb{R}^{n} \backslash\{a\} \mid \angle\left(x-a, e_{n}\right) \leq \alpha_{k}\right\}
$$

and let

$$
X_{k}:=A \backslash \operatorname{int} C_{k} .
$$

Then, evidently, $X_{k} \in \mathcal{S}^{n} \backslash \mathcal{A}$ for every $k$, and $\lim _{H} X_{k}=A$.

Further, let us notice that $\mathcal{A}$ is closed in $\left(\mathcal{S}^{n}, \varrho_{H}\right)$; indeed, if a sequence $\left(Y_{k}\right)_{k \in \mathbb{N}}$ in $\mathcal{A}$ is Hausdorff convergent to a star body $Y_{0} \in \mathcal{S}^{n}$, then, by $(2.2)$ applied twice, first to each $Y_{k}$ for $k \in \mathbb{N}$ (the implication $\Rightarrow$ ) and next to $Y_{0}$ (the implication $\Leftarrow$ ), we infer that $Y_{0} \in \mathcal{A}$.

Hence, $\mathcal{A}$ is nowhere dense in the space $\left(\mathcal{S}^{n}, \varrho_{H}\right)$ and so it is meager.

This completes the proof.

\section{Properties of some families of star bodies, fractal or nonfrac-}

tal. Let us start with Bloch's paper [1]. The author deals with any compact $n$-dimensional $C^{1}$-manifold with boundary, $M \subset \mathbb{R}^{n}$, and a $C^{1}$-embedding $f:$ bd $M \times[0, \infty) \rightarrow M\left(\right.$ or $f:$ bd $M \times[0, \infty) \rightarrow \mathbb{R}^{n} \backslash$ int $M$ ) satisfying condition $f(x, 0)=x$ for every $x \in \mathrm{bd} M$. Every continuous function $g: \operatorname{bd} M \rightarrow[0, \infty)$ determines a new manifold $G$ contained in $M$ (or containing $M$ ) such that bd $G$ is bilipschitz equivalent to $f$ (graph $g$ ). (Let us note that originally, instead of bilipschitz equivalence the author deals with some identifications.) Speaking more intuitively, when using $g$ one obtains such a new manifold by pushing bd $M$ inside (or outside) the given manifold $M$ along the unique fibres $f(\{x\} \times[0, \infty))$. The class of manifolds $G$ just described is denoted by $\mathcal{F}_{f}(M)$.

Let us observe that for some particular $M$ and $f$, the class $\mathcal{F}_{f}(M)$ coincides with $\mathcal{S}_{\varepsilon}^{n}$ defined in the Introduction.

REMARK 3.1. For a fixed $\varepsilon>0$, let $M:=\varepsilon B^{n}$, and let $f: \varepsilon S^{n-1} \times \mathbb{R}_{+}$ $\rightarrow \mathbb{R}^{n}$ be defined by

$$
f(\varepsilon u, t):=(\varepsilon+t) u \quad \text { for } u \in S^{n-1}, t \in \mathbb{R}_{+} .
$$

If $g \in C^{0}\left(\varepsilon S^{n-1} \times \mathbb{R}_{+}\right)$, then the function $\rho: S^{n-1} \rightarrow \mathbb{R}_{+}$defined by $\rho(u):=\varepsilon+g(\varepsilon u)$ is the radial function of a set $A \in \mathcal{F}_{f}(M)$ with

$$
\operatorname{bd} A=\left\{f(\varepsilon u, g(\varepsilon u)) \mid u \in S^{n-1}\right\} .
$$

This function $\rho$ is continuous and positive; moreover $\rho(u) \geq \varepsilon$ for every $u \in S^{n-1}$. Thus $A \in \mathcal{S}_{\varepsilon}^{n}$.

We are now going to prove two statements concerning arbitrary metric spaces. 
Proposition 3.2. Let $(X, d)$ be a metric space and let $\phi: X \rightarrow \mathbb{R}$ be a Lipschitz function. Then the function $\phi^{*}: X \times \mathbb{R} \rightarrow X \times \mathbb{R}$ defined by the formula

$$
\phi^{*}(x, \alpha(x)):=(x, \alpha(x)+\phi(x))
$$

is bilipschitz with respect to any product metric in $X \times \mathbb{R}$.

Proof. Consider the product metric $\bar{d}$ on $X \times \mathbb{R}$ :

$$
\bar{d}((x, \alpha),(y, \beta)):=d(x, y)+|\alpha-\beta| .
$$

It is metrically equivalent to any other product metric, (see [6] or [7, Lemma 2.2]).

Let $\lambda:=\operatorname{Lip} \phi$. Evidently,

$$
\lambda=\operatorname{Lip}(-\phi) \text { and }(-\phi)^{*}=\left(\phi^{*}\right)^{-1} .
$$

By (3.2) and (3.3),

$$
\begin{aligned}
\bar{d}\left(\phi^{*}(x, \alpha), \phi^{*}(y, \beta)\right) & =d(x, y)+|(\alpha+\phi(x))-(\beta+\phi(y))| \\
& \leq d(x, y)+|\phi(x)-\phi(y)|+|\alpha-\beta| \\
& \leq(1+\lambda)(d(x, y)+|\alpha-\beta| \\
& =(1+\lambda) \bar{d}((x, \alpha),(y, \beta)) .
\end{aligned}
$$

Thus $\operatorname{Lip}\left(\phi^{*}\right) \leq 1+\lambda$ and by $(3.4), \operatorname{Lip}\left(\left(\phi^{*}\right)^{-1}\right)=\operatorname{Lip}\left((-\phi)^{*}\right) \leq 1+\lambda$. This completes the proof.

Corollary 3.3. Let $(X, d)$ be a metric space and let $\phi: X \rightarrow \mathbb{R}$ be a Lipschitz function. Then for any function $\gamma: X \rightarrow \mathbb{R}$, the graphs of the functions $\gamma$ and $\gamma+\phi$ are bilipschitz equivalent.

Proof. Let us observe that the function $\phi^{*}$ defined by (3.2) maps graph $\gamma$ onto graph $(\gamma+\phi)$. Hence, $\phi^{*} \mid \operatorname{graph} \gamma$ is the required bilipschitz equivalence onto $\operatorname{graph}(\gamma+\phi)$.

We apply Corollary 3.3 to obtain the following modification of Theorem 4.4 of [7] concerning the Hausdorff dimension and a family of star bodies that is larger than $\mathcal{S}^{n}$.

Corollary 3.4. Let $A, L \in \mathcal{S}^{n}$ and let $\rho_{L}$ be Lipschitzian. Then for any fractal dimension $\operatorname{dim}_{F}$ and any nonempty $S_{0} \subset S^{n-1}$,

$$
\operatorname{dim}_{F}\left(\operatorname{graph}\left(\rho_{A \tilde{+} L} \mid S_{0}\right)\right)=\operatorname{dim}_{F}\left(\operatorname{graph}\left(\rho_{A} \mid S_{0}\right)\right) .
$$

Proof. Let $X:=S_{0} \subset S^{n-1}, \phi:=\rho_{A} \mid S_{0}$, and $\gamma:=\rho_{L} \mid S_{0}$. Then, by Corollary 3.3, the graphs of $\rho_{A} \mid S_{0}$ and $\rho_{A \tilde{+} L} \mid S_{0}$ are bilipschitz equivalent, whence the equality (3.5) holds.

Since any fractal dimension of the boundary of a star body in $\mathcal{S}^{n}$ equals the dimension of the graph of its radial function (see [7, Corollary 2.5]), as a direct consequence of Corollary 3.4 we obtain the following. 
Corollary 3.5. Let $\operatorname{dim}_{F}$ be any fractal dimension and let $L \in \mathcal{S}^{n}$ have the radial function Lipschitzian. Then the operation $A \mapsto A \tilde{+} L$ on $\mathcal{S}^{n}$ preserves the following subfamilies of $\mathcal{S}^{n}$ :

- the family of star bodies fractal with respect to $\operatorname{dim}_{F}$;

- the family of star bodies locally fractal with respect to $\operatorname{dim}_{F}$;

- the family $\left\{A \in \mathcal{S}^{n} \mid \operatorname{dim}_{F} \operatorname{bd} A=s\right\}$ for a given real $s \geq n-1$.

We are now interested in dense subfamilies of $\mathcal{S}_{\varepsilon}^{n}$ or $\mathcal{S}^{n}$.

Theorem 3.6. Let $A \in \mathcal{S}^{n}$ and $\varepsilon>0$. Then for any fractal dimension $\operatorname{dim}_{F}$, the family

$\mathcal{X}:=\left\{X \in \mathcal{S}_{\varepsilon}^{n} \mid\right.$ for every nonempty $S_{0} \subset S^{n-1}$,

$$
\left.\operatorname{dim}_{F} \operatorname{graph}\left(\rho_{X} \mid S_{0}\right)=\operatorname{dim}_{F} \operatorname{graph}\left(\rho_{A} \mid S_{0}\right)\right\}
$$

is dense in $\mathcal{S}_{\varepsilon}^{n}$.

Proof. Let $C \in \mathcal{S}_{\varepsilon}^{n}$ and $\alpha>0$. It suffices to find $X \in \mathcal{X}$ such that

$$
\sup _{u}\left|\rho_{X}(u)-\rho_{C}(u)\right| \leq \alpha .
$$

Since $\rho_{C}$ (being a nonnegative, continuous, and bounded function on $\left.S^{n-1}\right)$ is the restriction of a continuous, nonnegative, and bounded function on the ball $B^{n}$ (compare [2, (2.18)]), it can be approximated (with respect to the sup metric) by Lipschitz functions (for instance, by restrictions of polynomials in $n$ variables defined on $\left.B^{n}\right)$. Thus, there is a Lipschitz function $\phi: S^{n-1} \rightarrow \mathbb{R}_{+}$such that $\sup _{u \in S^{n-1}}\left|\phi(u)-\rho_{C}(u)\right|<\alpha / 3$.

Let $X$ be defined by

$$
\rho_{X}(u):=\phi(u)+\frac{\alpha}{3}\left(1+\frac{\rho_{A}(u)}{\sup \rho_{A}}\right) .
$$

Then $X \in \mathcal{S}_{\varepsilon}^{n}$, because for every $u \in S^{n-1}$,

$$
\rho_{X}(u)>\phi(u)+\frac{\alpha}{3}>\left(\rho_{C}(u)-\frac{\alpha}{3}\right)+\frac{\alpha}{3}=\rho_{C}(u) \geq \varepsilon .
$$

Further, for every $u \in S^{n-1}$,

$$
\begin{aligned}
\left|\rho_{X}(u)-\rho_{C}(u)\right| & =\left|\phi(u)+\frac{\alpha}{3}\left(1+\frac{\rho_{A}(u)}{\sup \rho_{A}}\right)-\rho_{C}(u)\right| \\
& \leq\left|\phi(u)-\rho_{C}(u)\right|+\frac{\alpha}{3}\left(1+\frac{\rho_{A}(u)}{\sup \rho_{A}}\right) \leq \alpha .
\end{aligned}
$$

Finally, let $\emptyset \neq S_{0} \subset S^{n-1}$. Then from Corollary 3.4 it follows that

$$
\operatorname{dim}_{F} \operatorname{graph}\left(\rho_{X} \mid S_{0}\right)=\operatorname{dim}_{F} \operatorname{graph}\left(\rho_{A} \mid S_{0}\right) ;
$$

hence $X \in \mathcal{X}$.

This completes the proof. 
The following remark can be easily generalized to arbitrary topological spaces and unions of their subspaces. We formulate it for our particular case only.

Remark 3.7. Let $\mathcal{A}$ be a subfamily of $\mathcal{S}^{n}$. If for every $\varepsilon>0$ the intersection $\mathcal{A} \cap \mathcal{S}_{\varepsilon}^{n}$ is dense in $\mathcal{S}_{\varepsilon}^{n}$, then $\mathcal{A}$ is dense in $\mathcal{S}^{n}$. (This is a direct consequence of the fact that the closure of a union contains the union of the closures.)

As a direct consequence of Theorem 3.6 we obtain the following.

COROLlary 3.8. Let $\operatorname{dim}_{F}$ be a fractal dimension and let $s$ be a real number, $s \geq n-1$. Then each of the following subfamilies of $\mathcal{S}^{n}$ is either empty or dense in $\mathcal{S}^{n}$ :

- $\left\{X \in \mathcal{S}^{n} \mid \operatorname{dim}_{F}\right.$ bd $\left.X=s\right\}$;

- $\left\{X \in \mathcal{S}^{n} \mid \forall U \subset\right.$ bd $X, U$ is nonempty and open in bd $X \Rightarrow \operatorname{dim}_{F} U$ $=s\}$

- $\left\{X \in \mathcal{S}^{n} \mid X\right.$ is locally fractal with respect to $\left.\operatorname{dim}_{F}\right\}$.

Corollary 3.9. Both the family $\mathcal{F S}^{n}\left(\operatorname{dim}_{F}\right)$ of star bodies fractal with respect to $\operatorname{dim}_{F}$ and its complement are dense in $\mathcal{S}^{n}$.

Proof. Applying Corollary 4.8 for any $s>n-1$ we obtain the first part of the statement. For $s=n-1$ we obtain the second part.

Acknowledgments. We are grateful to Elżbieta Pol for a helpful discussion.

\section{References}

[1] W. L. Bloch, Fractal boundaries are not typical, Topology Appl. 154 (2007), 533-539.

[2] K. Borsuk, Theory of Retracts, Polish Sci. Publ., 1967.

[3] P. M. Gruber, In most cases approximation is irregular, Rend. Sem. Mat. Univ. Politec. Torino 41 (1983), 9-33.

[4] - Dimension and structure of typical convex sets, continua and curves, Monatsh. Math. 108 (1989), 149-164.

[5] P. Goodey and W. Weil, Intersection bodies and ellipsoids, Mathematika 42 (1995), 295-304.

[6] I. Herburt and M. Moszyńska, On metric products, Colloq. Math. 62 (1991), 121133.

[7] I. Herburt, M. Moszyńska and D. Pronk, Fractal star bodies, in: Convex and Fractal Geometry, Banach Center Publ. 84, Inst. Math., Polish Acad. Sci., 2009, 149-171.

[8] M. Moszyńska, Selected Topics in Convex Geometry, Birkhäuser, 2006.

[9] R. Schneider, Convex Bodies: the Brunn-Minkowski Theory, Cambridge Univ. Press, 1993. 
[10] T. Zamfirescu, Description of most starshaped surfaces, Math. Proc. Cambridge Philos. Soc. 106 (1989), 245-251.

Maria Moszyńska

Grzegorz Sójka

Institute of Mathematics

University of Warsaw

Department of Mathematics and Information Sciences

Banacha 2

Warsaw University of Technology

02-097 Warszawa, Poland

Pl. Politechniki 1

E-mail: mariamos@mimuw.edu.pl

00-601 Warszawa, Poland

E-mail: sojkag@mini.pw.edu.pl

Received December 8, 2009 